\title{
PRÁCTICAS DEL PAISAJISMO FRANCÉS: LA CONSTRUCCIÓN DEL PROYECTO DEL PAISAJE EN EL PLAN TURÍSTICO MASPA- LOMAS COSTA CANARIA. 1961
}

Iván ÁLVAREZ LEÓN

El estudio de las prácticas que define el panorama efervescente del paisajismo francés durante los años 60, están representandas y defendidas por los elementos que explican la construcción del plan turístico de Maspalomas Costa Canaria de 1961. La vinculación directa que posee el plano y los miembros del atelier AS (autores del plan) con las intervenciones urbanas y paisajísticas en Francia, se convierten en el principal argumento de esta investigación.

Durante los años 60 en la región metropolitana de París se realizaron las mayores transformaciones del paisaje urbano y rural, a través de las políticas urbanas denominadas grand ensembles. De las soluciones empleadas en estas operaciones y del nuevo estado emergente del paisajismo en el diseño de planes, analizaremos la vinculación que existe con algunas de las soluciones planteadas en el plan turístico de Maspalomas.

Paisaje turístico, atelier AS ATEA+SETAP, proyecto Maspalomas Costa Canaria, urbanismo francés, Grand ensembles.

\section{FRENCH URBANISM PRACTICES: THE CONSTRUCTION OF THE LANDSCAPE PROJECT IN THE TOURISTIC PLAN MASPALOMAS COSTA CANARIA, 1961}

The Maspalomas Costa Canaria master plan designed in 1961 represented the beginning of a new urban practice in the European tourism planning based on the landscape as principle of urban design.

Over the 60s were developed the most important urban and rural landscape operations in the metropolitan area of Paris. The grand ensembles based on housing plans were promoted by the state urban policy in order to close the existing housing deficit in the dense cities of France. During 60s a new framework in the landscape operations and the solutions used to develop the grand ensembles were the keys to design the Maspalomas Costa Canaria master plan as one of the firsts advanced tourism plans in Europe. The main goal of this research pretends to highlight a strong correlation among the Maspalomas master plan, the AS atelier (architects of master plan) and the French landscape vision.

Touristic landscape, atelier AS ATEA+SETAP, Maspalomas project, Costa Canaria, French urbanism, Grand ensembles. 


\section{Introducción}

El presente trabajo de investigación desvela el carácter singular y avanzado del plano ganador en el concurso internacional de ideas Maspalomas Costa Canaria (1961). Algunas de las características del plano elaborado hace 50 ańos por el atelier francés AS, hoy, se han convertido en principios de ordenación para los desarrollos turísticos en la costa.

Maspalomas, pionera entre los desarrollos turísticos de costa en Europa, contribuye a través del primer plan de ordenación (no realizado) al pasado y futuro de los centros turísticos. La investigación ha incidido en los valores que el propio despacho de arquitectos anunció aquel año, como son el paisaje y el respeto por lo "natural".

El proyecto diseñado por el atelier francés AS ATEA+SETAP, objeto del concurso, presentó cualidades y características prodigiosas para su época ${ }^{1}$. Hoy, 50 años después se convierte en uno de los planes destacados del resto de las intervenciones turísticas proyectadas durante los años 60 del litoral español y francés.

El autor de la investigación para entender algunos de los elementos del plano que definen su carácter singular y avanzado, ha estudiado su expresión más evidente, como es el paisaje y su momento de cambio en Francia durante los ańos 60.

\section{Del paisaje subordinado en la ciudad al territorio como paisaje activador}

Algunas de las operaciones turísticas que se realizaron durante los años 60 en la región de Languedoc-Roussillon y en la franja de la Côte d'Azur, mostraron una nueva visión del paisaje que dio forma al plan de Maspalomas. En el proyecto de AS podemos identificar un modo de trabajar con el paisaje que presentan también algunos proyectos turísticos del sur de Francia.

Para ello, fue necesario entender cómo el paisaje era planificado y quienes eran los encargados para hacerlo. El paisaje en estas operaciones subordinadas por el sector público podía ser ordenado en dos categorías, según la clase de uso y escala: 


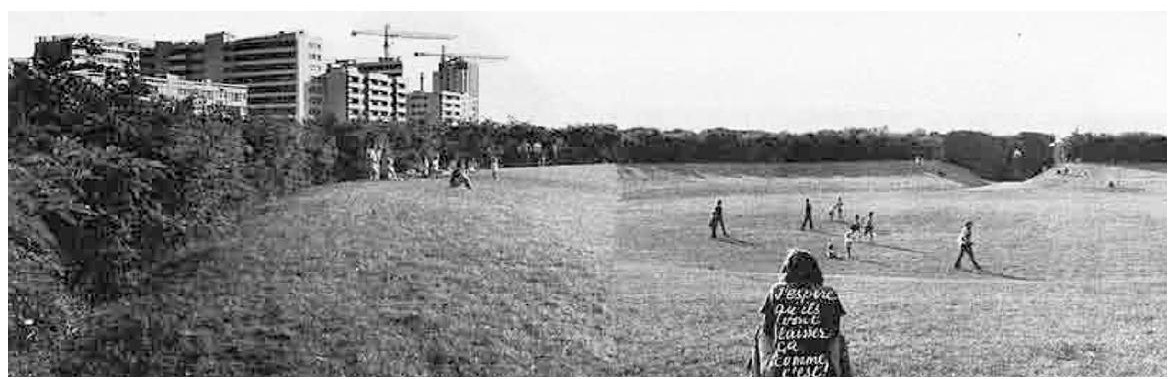

Fig. 1. Imagen del Paisaje como zonas protegidas del territorio en el Parque Saint-John-Perse, Reims. Fuente: Blanchon B., 1999, p.28.

1. Espacios Verdes Urbanos. La ciudad debía de ofrecer una variedad en los espacios verdes urbanos, con la finalidad de satisfacer las funciones de entretenimiento: juegos, deportes al ciudadano, etc.

2. Paisaje. El territorio debía planificarse con áreas naturales de protección o reserva, con la finalidad de mantener un paisaje con identidad a escala regional.

Estas dos líneas de acción simplifican cuales fueron los objetivos de la planificación para adaptarse armoniosamente a los paisajes rurales y naturales (territorio), con las estructuras económicas y humanas (ciudad).

El paisajista fue la figura encargada de dar forma al proyecto del paisaje en el ámbito de la planificación. No fue hasta 1960 cuando vino reconocida la especialidad de Paysage et de l'Art des Jardins' ${ }^{2}$ y poder ejercer como tal. Hasta entonces fueron los ingenieros, arquitectos y urbanistas quienes reflexionaron sobre el término paisaje y sus derivaciones, poniéndolo en práctica en proyectos del sector público y privado.

La reciente creación de la figura del paisajista durante el periodo de regeneración y expansión de la ciudad de París (como necesidad de incorporar una nueva visión en la escena urbana), no implicó la inmediata participación en los planes que se estaban llevando a cabo. La nueva formación en la enseñanza y su imprecisión en el termino paisaje, supuso que se cuestionara su práctica. El paisajista francés Jacques Simon manifestó su inquietud en el contexto cultural en el que se encontraban, con la siguiente declaración:

Qu'est-ce qu'un paysagiste? reste encore plus ou moins indéterminée, puisque c'est toujours en termes vagues que l'on aborde ce sujet. ${ }^{3}$

2 El decreto del 27 agosto de 1946 estableció la creación de la "sección" de Paysage et Arts des Jardins, dentro de la Enseñanza Superior Agrícola, y en particular e l'Ecole Nationale Supérieure d'Horticulture.

En el año 1960 con el decreto 61-632 del 20 junio 1960, será cuando se admita por primera vez en Francia dentro de la Enseñanza Superior Agrícola la "especialidad" de Paysage et Arts des Jardins, ejerciendo así como Paisajistas D.P.L.G (diplômé par le gouvernement) del Estado francés.

3 Simon J, 1964, p.131. 
La indeterminación de la figura del paisajista planteó una rápida respuesta en la definición del término ¿qué es paisaje? La oportunidad de establecer conceptos claros al término paisaje, sirvió para impulsar a los grupos de atelier sus nuevas capacidades en la ordenación. Y de esta manera, asumir a paisajistas como profesionales vinculados a los programas de planificación.

El plan de Maspalomas se diseñó en medio de este panorama de indefinición. Entre la creación de una nueva especialidad de paisaje en la enseñanza y la elaboración de pautas para proyectarlo. Frente a esta situación Maspalomas supuso una respuesta singular, con un proyecto que destacó por su fuerte vinculación con lo existente y el diseño de un paisaje territorial como estructura ordenadora del plan.

El proyecto representó un ejemplo para evidenciar cuales debían ser aquellas pautas que defina el término. Algunos de los atelier años más tarde (André Gutton, Guillaume Gillet) continuaron poniendo en práctica las características empleadas en Maspalomas, reforzando el término proyecto del paisaje.

El urbanismo durante años había empleado el término paisaje como espacio vacío, con la peculiaridad de dotar banalmente al espacio público de vegetación ${ }^{4}$. El plan turístico de Maspalomas se opone a esta concepción y defiende la postura más crítica de Simon J. hacia un paisaje activador. Un espacio que se distancia del concepto "vacío o complementario", para acercarse al de "ordenador y estructurador".

Este nueva concepción del espacio se manifestó en el plan como un espacio verde dinámico y continuo, características no estáticas que definen el carácter de la naturaleza. Algunos de los planes turísticos de este periodo destacaron por la nueva escena urbana proyectada entre el espacio verde y la arquitectura.

Proyectos como los del urbanista Jean Balladur en La Grande Motte reemplazaban la coreografía urbana de los Grand ensembles de París, por la interpretación de un paisaje de mayor movimiento y libertad para el ciudadano.

La dificultad del arquitecto en abstraer elementos de la naturaleza intangibles, determinaba la mayor o menor rigidez del proyecto (climatología, paisajes sensoriales, etc). Aquellos profesionales más ortodoxos y formalistas eran impulsados por el azar y la improvisación. En ese momento era cuando el paisajista debía desarrollar su labor como proyectista, adaptando su visión de paisaje al proyecto. A través del conocimiento de los fenómenos naturales, y no mediante la imposición de dispositivos preconcebidos en la ciudad, el paisajista podía establecer nuevos imputs a los planes turísticos.

El atelier AS en la propuesta para Maspalomas reflexionó en la adaptación de algunos de los elementos que los paisajistas defendieron para la ordena- 
ción del territorio, como fueron: ordenación de vegetación homogénea a la preexistente; adaptación a la topografía que daba forma al paisaje y el relieve; y estudio de las zonas de riesgo según los sistemas geológicos e hídricos del territorio.

El respeto por la topografía, las diferentes densidades de vegetación y la conservación de la zona de dunas mediante la implantación de usos ligeros, fueron algunos de los elementos que posicionó el plan dentro de los parámetros que el paisajismo francés defendió durante los años 60 .

Hasta ahora hemos explicado como la creación de una nueva especialidad en la enseñanza (paisajismo) impulsó la reflexión sobre la misión del paisajista frente al proyecto urbano, alcanzando una situación de emergencia profesional en la disciplina. Sin embargo, esta situación de imprecisión sobre ¿que es un paisajista? derivó a cuestionarse sobre ¿que es paisaje?. La ordenación de los nuevos centros turísticos fueron los encargados de dar respuesta a esta situación, con proyectos que ponían en valor la capacidad activadora y ordenadora del paisaje.

Las intervenciones diseñadas por los atelier y sus equipos multidisciplinares tuvieron como precedente el plano del atelier AS ATEA+SETAP proyectado para el concurso de Maspalomas en 1961. Dos de las características que empleaba el atelier AS en sus proyectos, como respuesta al término paisaje, fue el cambio de escala y continuidad.

\section{a. Cambios de escala}

La ordenación del paisaje y los cambios de escala en la proyectación iban a transformar la estructura espacial del territorio. Desde la escala urbana, los centros turísticos se encargaron de interpretar el paisaje a través del diseño de los espacios verdes, como ocurrió en la ciudad de París ${ }^{5}$. Y desde el territorio, los nuevos planes territoriales comenzaron a introducir áreas de bosque y zonas agrícolas como sistemas de protección y de reserva del paisaje.

Los planes directores del atelier AS se proyectaron introduciendo en el territorio las zonas de espacios verdes que la ciudad debía mantener, y las áreas de bosque que tenían que proteger. La visión de tener que reposicionar el carácter del paisaje, se manifestaba en la preocupación de entender el territorio desde todos los elementos que lo componían. El plan de Toulon elaborado en 1961 representó claramente un modelo de territorio delimitado por la práctica del zonning, además de ordenar áreas dispersas y discontinuas de bosque. Estas zonas naturales con una actividad agrícola fueron preservadas como economía local según las características del plan. 


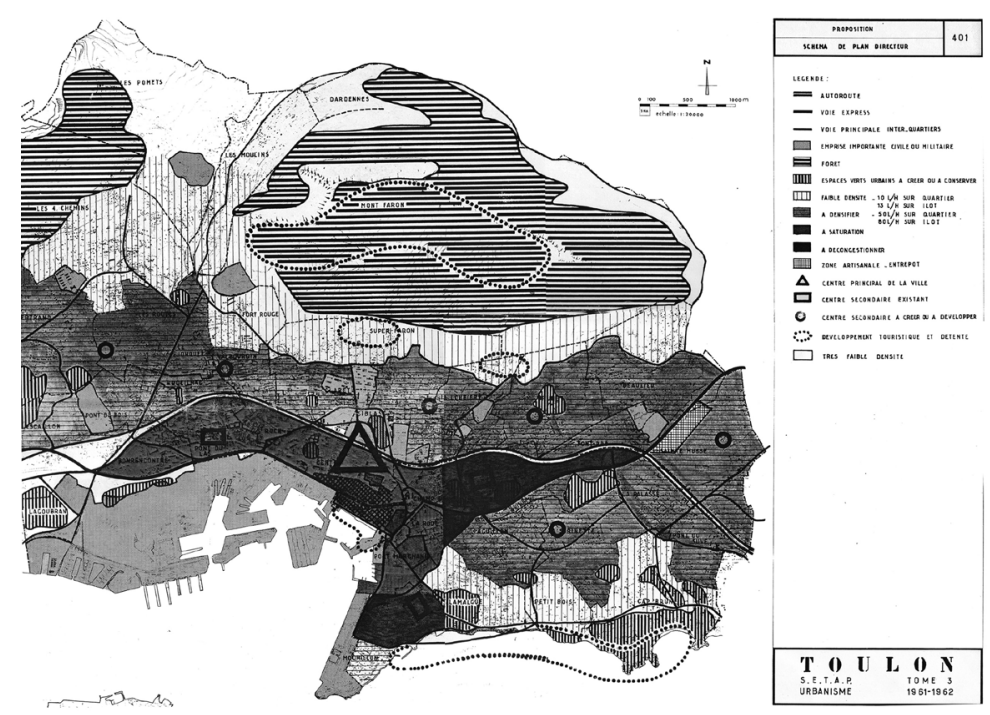

Fig. 2. Plan de Toulon, representación del concepto Paisaje-Territorio. Atelier SETAP. 1961-1962. Fuente: Centre de documentation de l'urbanisme, París.

\section{b. Continuidad}

Una de las principales peculiaridades del nuevo paisaje implícito fue la continuidad de espacios entre la arquitectura, la ciudad y el territorio, o la disolución de escala que existía entre lo doméstico, el urbano y el territorio. La experiencia del atelier AS para la nueva ciudad de Cansado ${ }^{6}$ articuló de manera jerárquica la tipología arquitectónica con los espacios públicos (calles y plazas), y estos a su vez con la topografía, climatología y modo de vivir ${ }^{7}$. El plan contribuyó a dar forma a la ciudad mediante uno de los conceptos empleados por el movimiento moderno, como fue la disolución del paisaje continuo.

En esta nueva situación por encontrar en la ciudad y el territorio un paisaje escalado y continuo, heterogéneo y estimulante, es precisamente donde el plan de Maspalomas se posiciona como protagonista. Un proyecto que fue capaz de reflejar en el dibujo los paisajes implícitos que se generan entre la ciudad y el territorio.

6 La ciudad de nueva creación de Cansado (Mauritania) fue uno de los primeros proyectos urbanos que realizó el atelier AS. Algunos conceptos como es el desarrollo secuencial de "unidades urbanas" fueron posteriormente aplicados al proyecto de Maspalomas. Para la ciudad de Cansado se proyectaron 5 unidades de 5.000 hab. y los equipamientos necesarios para una población total de 35.000 hab. Además de entender el planeamiento como un sistema unitario que encontró respuestas en el propio territorio, las soluciones empleadas a escala arquitectónica por "agrupaciones", y territorial a través de "unidades urbanas", fueron el resultado de entender la ordenación como un sistema continuo y flexible.

7 Abram J, 1999, p.278. 


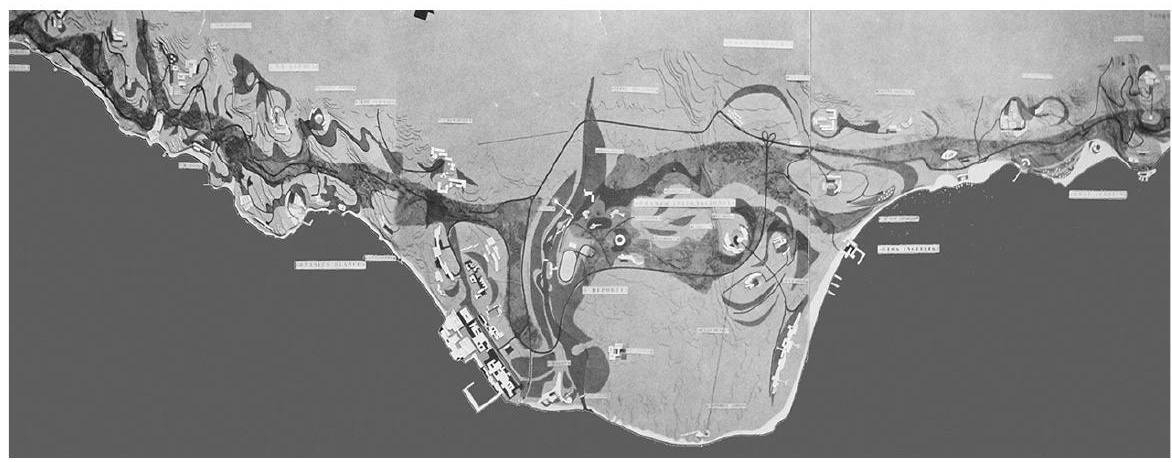

Fig. 3. Imagen 3. Plan de Maspalomas realizado por el atelier francés AS ATEA+SETAP. 1962. Fuente: Vega Grande Conde, 1963.

\section{Las políticas urbanas en Francia y la construcción del proyecto del paisaje}

El plan de Maspalomas representó una intervención territorial avanzada dentro del contexto del Planeamiento francés. La ordenación estructuró de manera imperiosa el paisaje y los asentamientos urbanos, reflejando las características más singulares del lugar.

La ruptura de las prácticas tradicionales en el urbanismo moderno de los años 50 y 60 localizadas en los centros urbanos y periferias de las ciudades, derivaban en ocasiones del paisaje y de las reflexiones que se estaban gestando en los desarrollos turísticos en la Costa Sur de Francia.

Los nuevos asentamiento turísticos de Languedoc-Roussillon y de la Côte d'Azur se convirtieron en laboratorios para experimentar entornos naturales con nuevas tipologías de habitación. Los atelier tuvieron la oportunidad de proyectar nuevas relaciones urbanas con el paisaje. La investigación posiciona el plan de Maspalomas como unos de los proyectos más paradigmáticos que representa la relación paisaje, ciudad y turismo.

\section{Los grands ensembles 1955-1973. Saltos de escala y yuxtaposición de pai- sajes en el plano de Maspalomas.}

Los grands ensembles se trataban de operaciones urbanas localizadas en los límites de la ciudad, con el objetivo de construir una nueva zona residencial capaz de descongestionar el centro y solucionar las carencias del parque inmobiliario. En 1948 comenzaron las primeras intervenciones con operaciones que no superaban las 100 unidades residenciales. Siete años más tarde, a mitad de los años 50, alcanzaron la cifra de hasta 10.000 unidades en cada operación urbana ${ }^{8}$.

8 Este cambio de escala fue posible gracias a los esfuerzos y avances técnicos de los elementos prefabricados empleados en la arquitectura. Se pasó de la construcción mediante métodos artesanales a la producción industrial, 
La escala de este tipo de proyectos fue de tales dimensiones que en ocasiones transformaba la estructura urbana de la ciudad, afectando a las infraestructuras viarias existentes y desplazando las áreas productivas a otras zonas del territorio.

Debido a los desequilibrios sociales que se originó en los centros urbanos, este tipo de políticas adoptadas por las administraciones públicas recibieron críticas de célebres arquitectos y urbanistas? ${ }^{9}$ La construcción masiva y dispersa de las intervenciones hizo que fueran denominadas por algunos críticos de grandes "islas urbanas"10, con poblaciones que alcanzan hasta los 50.000 habitantes.

Los grands ensembles aunque se trataran de operaciones con falta de referencia urbana y conexión con la estructura general del territorio, el espacio interior de la "isla urbana" establece una serie de prácticas de interés para la investigación. El estudio refleja la relación entre arquitectura y espacio verde, como un nuevo concepto de paisaje y modo de vivir en la ciudad.

Algunos ejemplos como Marly Les Grands Terres en 1957-1957 del arquitecto Marcel Lods o Patin-Les Courtilières ${ }^{11}$ en 1955-1959 de Emilie Aillaud, dieron forma al espacio central como amplios espacios verdes. Las diferentes escalas que adquiere el espacio y su relación con el edificio (espacio verde central y espacio entre unidades residenciales) dieron orden y estructura a los nuevos paisajes urbanos que estudiaremos.

Estas intervenciones comparables a las new-towns inglesas o barrio holandés de 40.000 y 50.000 habitantes $^{12}$, distribuían la superficie destinada a los espacios verdes públicos en un 35\% del total de la superficie. En el caso de Marly el espacio verde estaba constituido por nueve unidades de iguales dimensiones y tres edificios que gravitaban entorno a él. Este espacio se encargaba de recoger todas las funciones de la vida urbana, como equipamientos deportivos y juegos colectivos. La arquitectura empleada fue considerada como un ejemplo sumamente racional que proporciona a todas las unidades de alojamiento las mismas condiciones de habitabilidad.

El proyecto de Les Courtilières ocupa una superficie de 17 hectáreas, de las cuales 5 estaban ocupadas por el espacio central. Este espacio está configurado por la fachada continua de un edificio con más de un kilómetro de longitud. En su interior se generaban las reflexiones sobre cómo el paisaje podía contribuir a un nuevo modo de vivir la ciudad. Un cambio de direc-

bajando de esta manera considerablemente los costes y las horas de construcción de una vivienda.

9 André Bloc en la revista L'architecture d'Aujourd'hui. (1962) manifestó su desacuerdo con este tipo de sistemas declarando que "...salvo algunas excepciones, han creado ciudades sin alma y sin interés arquitectónico. En el conjunto urbano de la región parisina se han ido desparramando los grandes complejos, sin más justificación que la existencia de terrenos disponibles". No obstante, considera que es una buena oportunidad para transformarlos en nuevos centros urbanos dotándoles de equipamientos y servicios que abastezcan la población de los grands ensembles.

10 Piccinato G, 1969, p.154.

11 El proyecto representa uno de los ejemplos mas emblemáticos de los grands ensembles de aquellos años. En 1960 ganó el Grand Prix 1960 du Cercle d'études architecturales

12 Idem, p. 140. 


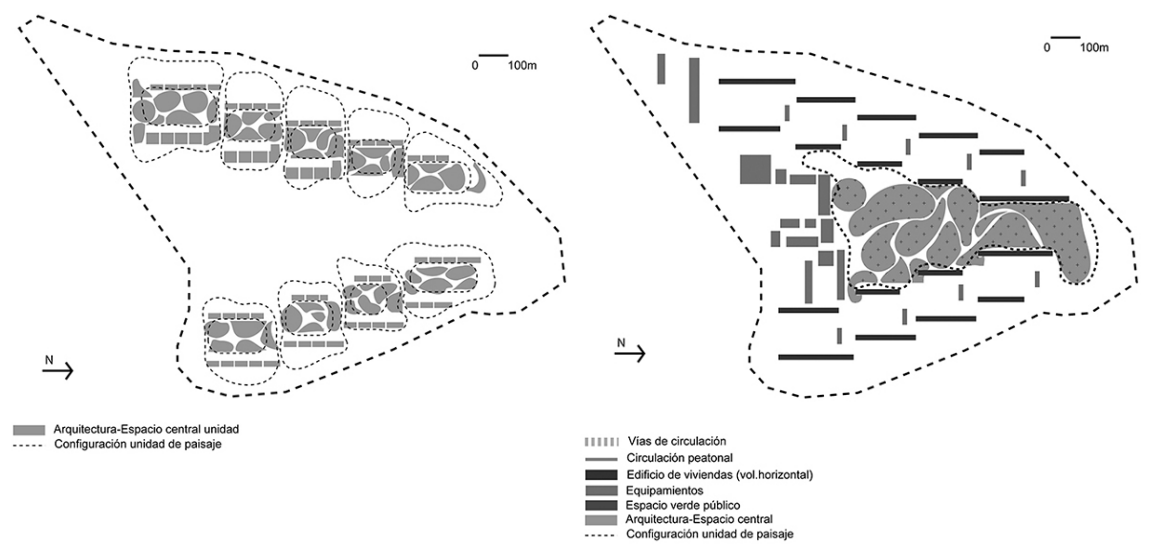

Fig. 4. Esquemas de la vegetación utilizada en el espacio central y unidades de paisaje del grand ensemble Les Grandes-Terres. Marly-le-Roi. Arq. Marcel Lods. 1952-1960. Elaboración propia.

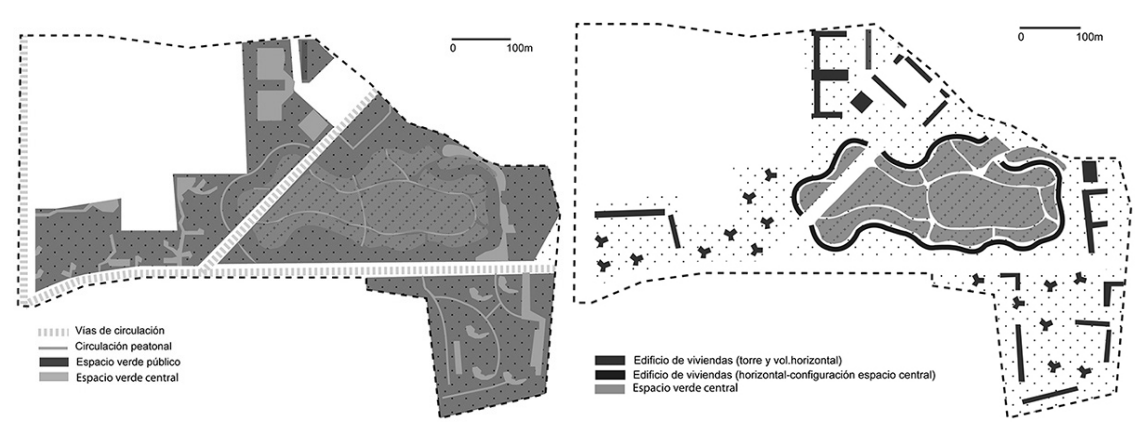

Fig. 5. Esquemas de la red de caminos en el espacio verde público y configuración del espacio central del ensemble Les Courtillières. Arq. Emile Aillaud. 1955-1965. Elaboración propia.

ción en el diseño de estos espacios verdes convirtió el espacio central de los grands ensembles en espacios privilegiados de la ciudad. Estos espacios se caracterizaron por la ordenación y modulación del terreno; disposición del arbolado; y creación de caminos. El paisaje proyectado en ocasiones adquiere la categoría de relieve topográfico, configurando de este modo un ambiente estimulante para la ciudad moderna.

El elevado número de viviendas y la estandarización de los elementos constructivos hizo que la calidad arquitectónica pasara a un segundo plano. Los esfuerzos de los atelier se concentraron en el diseño de los espacios libres, en la relación entre arquitectura y espacio público, y en los programas y usos urbanos.

La complejidad de los grands ensembles en las fases del diseño y construcción fue tal, que hizo que los atelier comenzaran a formar grupos de profesionales 
multidisciplinares. La figura del paisajista fue como anunciábamos anteriormente, una de las visiones singulares en este tipo de operaciones. El atelier AS estaba compuesto por arquitectos, urbanistas, paisajistas, abogados, y economistas con el propósito de realizar todos los aspectos de urbanización y arquitectura necesarios para desarrollar este tipo de planes.

La investigación desvela tres características del paisaje proyectado por algunos de los atelier multidisciplinares y de reconocimiento en Francia. Y donde la visión predominante del paisajista es evidente:

1. Capacidad ordenadora: los porcentajes de hasta un $35 \%$ del total de la superficie destinado a espacio verde público (exento de vías y aparcamientos) beneficia al diseño de amplios espacios libres capaces de configurar un sistema verde "estructurador" (ver imagen 6 y 7 ).

2. Unidades homogéneas: El empleo de agrupaciones de edificios formando unidades facilita la ordenación de estas grandes operaciones, racionalizando los costes y las condiciones ambientales de las viviendas. De esta solución surgen espacios verdes centrales entre unidades residenciales que incrementan el carácter de una comunidad (ver imagen 8).

3. Arquitectura-espacio central: La disposición de la arquitectura está configurada por el espacio central de un espacio verde abierto que viene delimitado por sus propias reglas (ver imagen 9 y 10 ).

Estas tres características que sintetizan la visión de la ciudad con el paisaje, son también puestas en práctica en algunas de las propuestas realizadas por el atelier AS ATEA+SETAP.

Entre las operaciones que elaboró AS de menor escala destacaron los ensembles de Les Buffets con 260 unidades (1959), Cergy Pontoise con 411 y 326 unidades (1969), y Trappes St Quentin con 200 unidades (1972). El ensemble de mayor escala corresponde a Cofl ants ste Honor con 3.000 viviendas y una población de 15.000 habitantes (1965). Pero fue en el plan de Maspalomas (1961) cuando trabajaron las soluciones que planteaba el paisaje de los grands ensembles de manera más evidente. A continuación se detalla las características de cada una de ellas y su vinculación en el plan turístico de Maspalomas.

\section{a. Capacidad organizadora urbana - territorial}

Con el cambio de escala de los grands ensembles el paisaje adquirió nuevas y numerosas formas en su organización espacial. En 1961 sobresalieron algunos de los trabajos realizados en la ENSBA durante el Seminario y Atelier Tony Garnier. Los proyectos de estudiantes dirigidos por los atelier de arquitectos y urbanistas nacionales e internacionales tuvieron como finalidad ordenar un sistema de parques continuos. Fue a través de la capacidad or- 
ganizadora del paisaje cómo delimitaron el crecimiento de los núcleos y la conexión con los centros más dispersos.

Seis años más tarde de la elaboración del plan de Maspalomas podemos encontrar en el concurso de Bordeaux-Nord, cómo los atelier emplearon el paisaje para ordenar y estructurar toda la operación urbana. Al concurso se inscribieron prestigiosos urbanistas como André Gutton y Guillame Gillet, diseñando proyectos donde el paisaje se convirtió en el instrumento de la escena urbana. Ambos compartieron reflexiones teóricas similares y defendieron la capacidad organizadora del paisaje tan solo contrastando formalismo en sus dibujos. La investigación sitúa esta experiencia como la primera en emplear la nueva escena contemporánea del paisaje, después de la experiencia del plan de Maspalomas en 1961.

\section{b. Unidades homogéneas}

El plano de Maspalomas es característico por la disposición de núcleos que se distribuyen por todo el territorio colonizando aquellas zonas de mayor interés, como son las cimas de los promontorios y las áreas más cercanas a la línea de costa. La ordenación basada en el uso de zoning se consideró como la más adecuada debido al relieve topográfico. Los planes compuestos por varias unidades intensificaban su relación con el paisaje por medio de un incremento en las áreas de espacios verdes. Estas unidades capaces de adaptarse a una organización dinámica y flexible, son las que el autor define como unidades homogéneas de paisaje ${ }^{13}$.

\section{c. Espacio central - arquitectura central}

La secuencia de diagramas representa un cambio en la configuración de las unidades urbanas estudiadas hasta el momento. En los grans ensembles el espacio público abierto quedaba delimitado por el espacio que se creaba entre las arquitecturas (véase imagen 5). Sin embargo, en Maspalomas la arquitectura invierte su condición de límite y pasa a una posición central de mayor privilegio (creando una separación a través de la vegetación proyectada). La posibilidad que la arquitectura no sea quien configure el espacio verde, convierte el paisaje en el único instrumento para poder hacerlo, adquiriendo mayor libertad en sus límites.

Un análisis de las características del paisaje en algunas intervenciones urbanas desarrolladas en Francia y en el plan de Maspalomas, ponen de relieve la transformación que se estaba gestando en la práctica del paisaje durante los años 60. La investigación desvela algunas de esas pautas para hacer entender el carácter singular y avanzado del plan turístico de Maspalomas.

13 Alvarez León, I \& Cappai, A., Tourism Research: An Interdisciplinary Perspective. p. 194-195, 2013. 

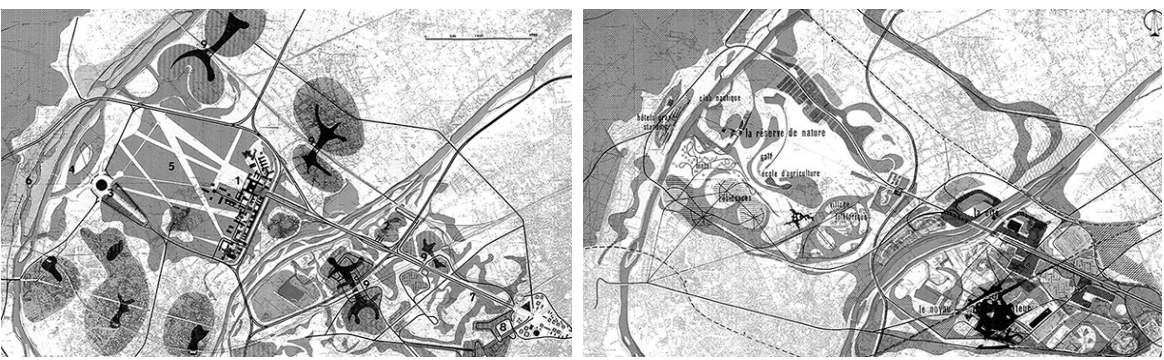

Fig. 6. Imagen del sistema de parques que estructura la región de La Defense. Seminario y Atelier Tony Garnie, 1961. Fuente: Urbanisme, 1964, 82-83, pp.103-115.

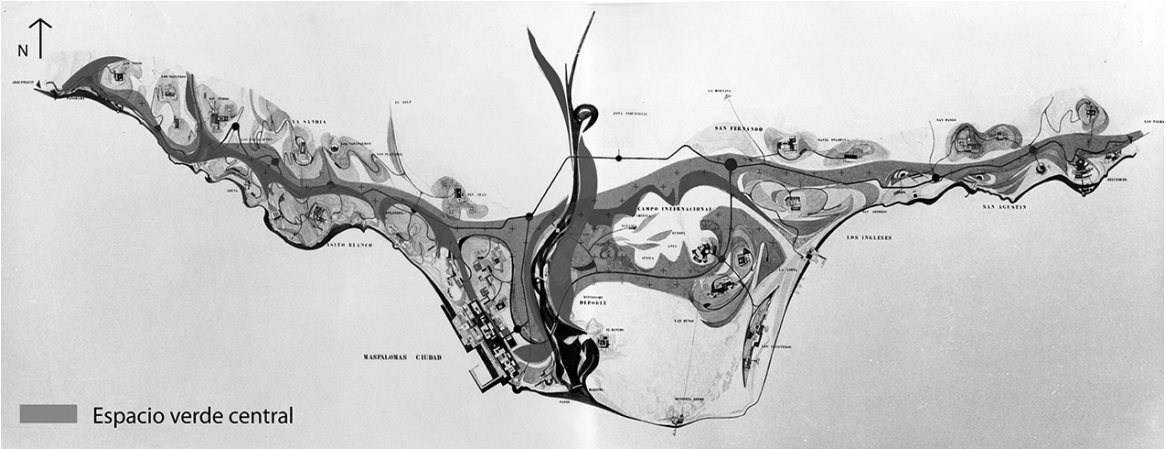

Fig. 7. Imagen del plano de Maspalomas. Esquema del sistema verde central como vertebrador del proyecto. 1962

๙

Unidades de paisaje y arquitectura

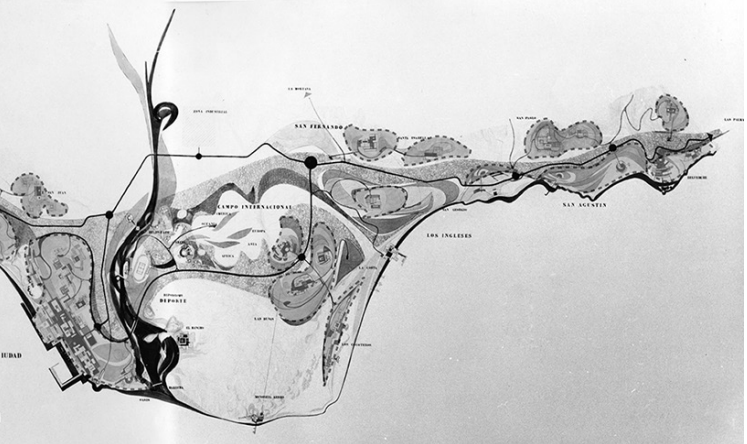

Fig. 8. Imagen del plano de Maspalomas. Esquema de las unidades de paisaje. 1962 


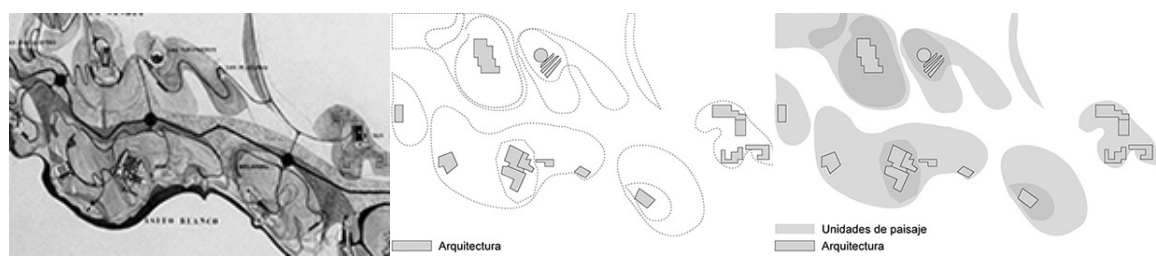

Fig.9. Esquemas de la configuración de las arquitecturas en las unidades de paisaje para el proyecto de Maspalomas. Atelier AS ATEA+SETAP. 1962
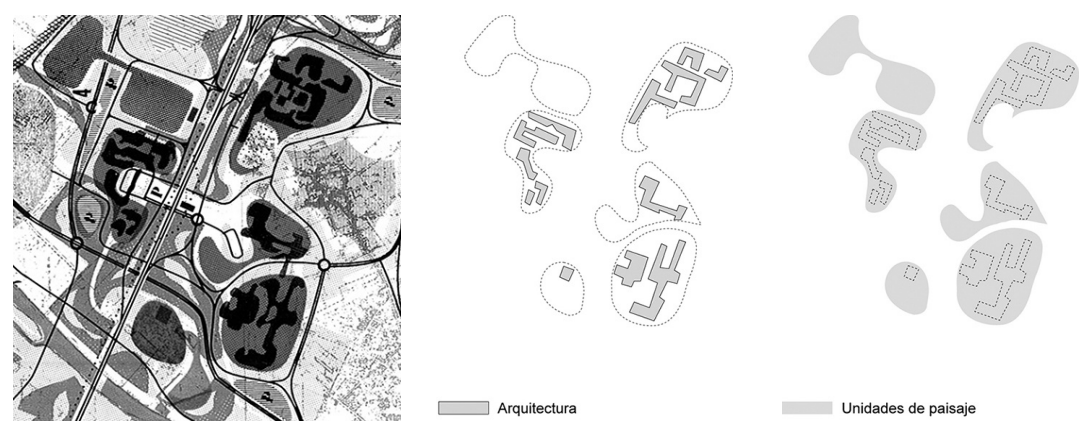

Fig.10. Esquemas de la configuración de las arquitecturas en las unidades de paisaje para el Seminario y Atelier Tony Garnier. Atelier Lemaresquier .1961

Es por ello, que en el cambio de una mirada subordinada del paisaje por una más activa, y en la práctica del espacio público de los grands ensembles, encontramos los elementos que construyeron el proyecto del paisaje del plan Maspalomas Costa Canaria.

\section{Conclusiones}

El plan turístico de Maspalomas de 1961 representa la práctica de un paisajismo (activador, dinámico y saltos de escala) como argumento principal en la planificación costera de los 60', haciendo de lo natural y de los elementos más físicos del territorio la base del proyecto urbano y turístico.

Un cambio en la concepción del paisaje a finales de los 50 sirve como línea de investigación para destacar el valor del paisaje en la planificación costera. Un paisaje que pasa de ser subordinado a activador del territorio, y donde los límites del espacio verde los establece la propia naturaleza y no la arquitectura.

Hoy, cincuenta años después el plan de Maspalomas Costa Canaria pertenece al reducido grupo de operaciones turísticas "cultas" en Europa de los años 60. Y donde el paisaje se convirtió por primera vez en sujeto "arquitecturas y unidades" y predicado "activador, escalar" del proyecto turístico. 


\section{Activador / Escalar / Continuidad}

El plan de Maspalomas aparece en medio de una situación de cambio en la enseñanza universitaria francesa, con la creación de la especialidad Paysage et Arts des jardins (paisajista). Esta nueva figura profesional plantea dos grandes interrogantes a principio de los ańos 60, ¿que es un paisajista? y ¿que es paisaje?. El plan de Maspalomas establece un punto de inflexión entre el termino tradicional de paisaje como jardín (escala) y fondo (escena urbana), para dar respuesta a una nueva concepción entendida como activador del territorio.

Maspalomas representa en 1962 la práctica de un planeamiento donde el paisaje se convierte en un sistema abierto, flexible y dinámico, introduciendo dos acciones extraordinarias dentro del contexto cultural en Francia, como fue:

a. Cambios de escala: La ordenación del paisaje iba a cambiar la estructura espacial de la ciudad por la del territorio. Un salto de escala hizo que Maspalomas trabajara con el paisaje para estructurar la escala global (territorio), y ordenar la local (urbana).

b. Continuidad escalar: Considerado como una derivación de la acción de los cambios de escala. El atelier elabora un plan donde el paisaje es el encargado de crear continuidad entre los espacios que surgen de las arquitecturas y núcleos urbanos (áreas verdes), y a su vez, entre el territorio y los asentamientos humanos (conservar las áreas de interés natural).

\section{Arquitecturas / Unidades homogéneas}

Las políticas urbanas empleadas en Francia a finales de los años 50 desencadenaron una serie de prácticas que transforma la forma urbana de la ciudad. $\mathrm{El}$ atelier AS manipula algunas de estas soluciones para configurar el paisaje de Maspalomas.

Los Grand ensembles. El espacio central (espacio verde público) de estas intervenciones se convierte en zonas privilegiados dentro de la ciudad. El atelier se basó en las características de ordenación de este espacio para introducir cuatro principios urbanos en el plan.

a. Capacidad ordenadora: Amplios espacios libres capaces de configurar un sistema verde "ordenador".

b. Arquitectura-espacio central: La arquitectura pasa de delimitar el espacio público a una posición central, donde el verde se delimita de manera natural y abierta. 
c. Unidades homogéneas: Agrupaciones de arquitecturas que forman unidades de asentamientos autónomos con la peculiaridad de interaccionar como un único sistema. El paisaje se configura del espacio de transición que surge entre unidades.

\section{Bibliografía}

Abram, J. (1999). L'architecture moderne en France : Du chaos à la croissance 1940-1966. vol. 2. Paris. Picard (Ed.). 273-278.

Alvarez León, I. (2011). The tourist project of the Setap group for the international bid of ideas for Maspalomas Costa Canaria. ARA Journal of Tourism Research, 3, 15-23.

Alvarez León, I \& Cappai, A., (2013). Tourist project of the SETAP Group for the International Bid of Ideas for Maspalomas Costa Canaria. Tourism Research: An Interdisciplinary Perspective. 191-206.

Blanchon, B. (1999). LES PAYSAGISTES FRANÇAIS DE 1945 À 1975 : L'OUVERTURE DES ESPACES URBAINS. Les annales de la recherche urbaine, 85. 21-29. [Libro en línea]: http:// www.annalesdelarechercheurbaine.fr/IMG/pdf/Blanchon_ARU_85.pdf

Piccinato, G. (1969). L'architettura contemporanea in Francia. Bologna. Cappelli (Ed.).154.

Pié, R. \& Barba, R. (Eds.), (1996). Arquitectura y Turismo: Planes y Proyectos. Barcelona. Universitat Politécnica de Catalunya-Plan Futures (Ed.).

Roncayolo, M. (1989), Paris en mouvement 1950-1985, en PARIS GENĖSE D'UN PAYSAGE. Bergeron, L. (coord). Picard (Ed.). 292-297.

Séminaire et atelier Tony Garnier: l'enseignement de l'urbanisme a l'Ecole Nationale Superiéure des Beaux-Arts. (1964), Urbanisme, 82-83, 99-115.

Simon, J. (1964). La mission du paysagiste: rubrique Espaces verts, Urbanisme, 82-83.

Vega Grande, Conde. (1963). Maspalomas Costa Canaria: Urbanización. Bilbao. Imprenta Industrial S.A. Bilbao (Ed.). 
Iván Álvarez León, Dr. Arquitecto

Departamento de Urbanismo y Ordenación del Territorio (DUOT)

Universidad Politécnica de Cataluña (UPC)

Instituto "Habitat, Turismo, Territorio"(UPC-UMA)

ial79arq@yahoo.com 